Check for updates

Cite this: J. Mater. Chem. A, 2018, 6 , 22145

Received 16th June 2018

Accepted 8th August 2018

DOI: $10.1039 / c 8 t a 05772 a$

rsc.li/materials-a

\section{Designing conjugated microporous polymers for visible light-promoted photocatalytic carbon- carbon double bond cleavage in aqueous medium $\uparrow$}

\begin{abstract}
Cyrine Ayed, ${ }^{\text {ab }}$ Lucas Caire da Silva, ${ }^{a}$ Di Wang ${ }^{a}$ and Kai A. I. Zhang (D) *a
The production of valuable carbonyl compounds directly from alkenes via carbon-carbon double $(C=C)$ bond cleavage is a highly important reaction. As a promising alternative to traditional metal-catalyzed thermal reaction conditions, photocatalysis offers an environmentally friendly and sustainable synthetic strategy. However, most photocatalysts used so far were homogeneous, molecular and transition metalbased catalytic systems. Here, we report a structural design strategy of conjugated microporous polymers (CMPs) as a class of metal-free, heterogeneous, stable and reusable photocatalysts for visible light-promoted $\mathrm{C}=\mathrm{C}$ bond cleavage reaction. Via different electron donor and acceptor combinations in the CMP backbone structure, we systematically investigated the structural influence of the CMPs on their optical and electrochemical properties, thereby revealing the structure and performance relationship in the photocatalytic $\mathrm{C}=\mathrm{C}$ bond cleavage reaction. As a result, the CMP with a specific electron donoracceptor combination containing benzothiazole as a strong electron withdrawing unit and phenyl as a weak electron donating unit was the most efficient photocatalyst. The $\mathrm{C}=\mathrm{C}$ bond in various alkenes, mostly styrene derivatives, could be successfully cleaved, forming aldehydes as a product with high conversion and moderate to high selectivity. The green aspect of the work is demonstrated by the utilization of visible-light, molecular oxygen as a cheap oxidant and water-based reaction medium.
\end{abstract}

\section{Introduction}

The synthesis of valuable carbonyl compounds such as aldehydes, acetic acid, acetaldehyde and ketone derivatives via carbon-carbon double $(\mathrm{C}=\mathrm{C})$ bond cleavage of alkenes is a highly important reaction in the chemical and pharmaceutical industries. These products have wide applications as important molecular building blocks and intermediates for the manufacture of perfumes, dyestuffs, agrochemicals and pharmaceuticals. $^{1-5}$ Various studies have reported the thermal catalytic oxidation of alkenes, notably styrene, for the desired carbonyl products. Important examples of metal catalysts are iron-based systems such as iron(II) chloride, ${ }^{6} \mathrm{Fe}\left(\mathrm{NO}_{3}\right) \cdot 9 \mathrm{H}_{2} \mathrm{O},{ }^{7}$ etc. ${ }^{8}$ Alternatively, Feng et al. have employed a water-soluble palladium(II) complex for the selective aerobic oxidation of styrene in water, obtaining benzaldehyde with high selectivity. ${ }^{9}$ Zhang et al. have also observed the $\mathrm{C}=\mathrm{C}$ bond cleavage and the formation of acetophenone with moderate selectivity over $\mathrm{ZrO}_{2}$-supported palladium nanoparticles. ${ }^{10}$ Recently, Liu et al. have reported the

${ }^{a}$ Max Planck Institute for Polymer Research, Ackermannweg 10, 55128 Mainz, Germany.E-mail: kai.zhang@mpip-mainz.mpg.de

${ }^{b}$ Graduate School for Excellence Materials Science in Mainz, Johannes Gutenberg University Mainz, Staudingerweg 9, 55128 Mainz, Germany

$\dagger$ Electronic supplementary information (ESI) available. See DOI: 10.1039/c8ta05772a use of gold nanoparticle mobilized carbon as a catalyst for the selective oxidation of styrene. ${ }^{2}$ The researchers pointed out that the strong Au-carbon interaction could enhance the catalytic efficiency.

In comparison to traditional thermal reaction conditions, photocatalysis offers an environmentally friendly and sustainable synthetic strategy. Nevertheless, there are only few photochemical reports on the photo-oxidative cleavage of the $\mathrm{C}=\mathrm{C}$ bonds of styrene. For instance, Guo et al. have reported the use of $\mathrm{Au}$-Ti-decorated mesoporous silica for the photo-epoxidation reaction of styrene with moderate selectivity. ${ }^{\mathbf{1 1}}$ More recently, Shen et al. have developed a chiral manganese catalyst to trigger the photocatalytic enantioselective epoxidation of terminal olefins, using water as an oxygen source, yielding epoxides with relatively high enantioselectivities. ${ }^{12}$ So far, the photocatalytic systems reported were mainly based on transition metals. Very few molecular organic photocatalysts have been developed, such as eosin Y, which was used as an organophotoredox to mediate the oxidative cleavage of the $\mathrm{C}=\mathrm{C}$ bond. ${ }^{13}$ However, despite the progress made so far in this field, considerable drawbacks are still associated with those metal based or organic molecular systems. For example, the toxicity of heavy metals, the instability and the lack of reusability of the molecular photocatalysts lead to the need for the development of a more stable, reusable, metal-free and heterogeneous photocatalytic system. 
Conjugated microporous polymers (CMPs), combining a visible light-active $\pi$-backbone and microporous properties, have recently emerged as a new class of efficient, stable and heterogeneous photocatalysts for chemical transformations. ${ }^{\mathbf{1 4 - 1 6}}$ Recent research activities demonstrated their wide application range in various photocatalytic reactions such as molecular oxygen activation, ${ }^{\mathbf{1 4 , 1 7 , 1 8}}$ selective oxidation of organic sulfides, ${ }^{19} \mathrm{C}-\mathrm{C}$ or $\mathrm{C}-\mathrm{N}$ bond formation, ${ }^{\mathbf{2 0 - 2 2}}$ dehalogenation, ${ }^{23,24}$ oxidative hydroxylation of arylboronic acids, ${ }^{25}$ visible light-initiated free radical and cationic polymerization, ${ }^{26,27}$ cycloaddition, ${ }^{28}$ oxidative homocoupling of amines, ${ }^{29} \mathrm{C}-3$ functionalization of indoles, ${ }^{30}$ and hydrogen evolution. ${ }^{31-34}$ So far, the photocatalytic oxidative $\mathrm{C}=\mathrm{C}$ bond cleavage using conjugated microporous polymers has not been reported.

Herein, we report the design of CMPs bearing different electron donor and acceptor-building blocks as heterogeneous photocatalysts for the $\mathrm{C}=\mathrm{C}$ bond cleavage of alkenes, mostly styrene derivatives. We investigated the structural influence of the CMPs on their photocatalytic efficiency. It was shown that the photocatalytic reaction could be conducted with almost quantitative conversion and selectivity using the CMP containing benzothiazole as a strong electron withdrawing unit, together with a phenyl group as an electron donating unit in the polymer backbone. Additionally, the use of aqueous reaction medium demonstrated its green and sustainable reaction nature. The mechanistic insight of the photoredox reaction was also investigated.

\section{Results and discussion}

In this work, for the structural design of the CMPs, we choose different building blocks according to their electron affinity. As displayed in Scheme 1, thiophene (Th) as a strong electron donor and benzothiodiazole (BT) as a strong electron acceptor were polymerized via the palladium-catalyzed Sonogashira cross-coupling reaction ${ }^{35}$ with triethynylbenzene (B) in the corresponding CMP backbone structures, referred to as BTh and BBT, respectively. A mixed CMP was also synthesized containing $50 \%$ BT unit and 50\% Th unit in the same manner, referred to as BThBT. The CMPs were obtained as amorphous solids as shown by the X-ray diffraction patterns (Fig. S1 $\dagger$ ). The detailed

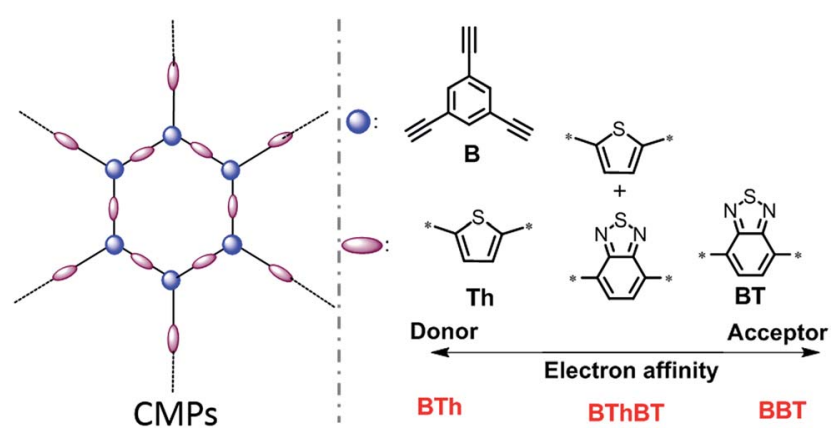

Scheme 1 Structural design of a series of conjugated microporous polymers (CMPs) via combination of different electron donor and acceptor building blocks. synthetic pathway with detailed characterization methods of the polymers are described in the Experimental section.

As displayed in Fig. 1, the scanning electron microscopy (SEM) images of the CMPs showed a variety of shapes. BTh was obtained as irregularly shaped particles with a size ranging between 40 and $180 \mathrm{~nm}$, whereas, BBT showed a fibrous structure with a diameter of $c a .200 \mathrm{~nm}$. BThBT appeared as fused large particles and fibers.

The Brunauer-Emmett-Teller (BET) surface areas of BTh, BThBT and BBT were measured to be $806 \mathrm{~m}^{2} \mathrm{~g}^{-1}, 445 \mathrm{~m}^{2} \mathrm{~g}^{-1}$ and $129 \mathrm{~m}^{2} \mathrm{~g}^{-1}$, with pore sizes of $1.7 \mathrm{~nm}, 1.41 \mathrm{~nm}$ and $1.7 \mathrm{~nm}$, respectively. The nitrogen gas sorption isotherms and pore size distributions are displayed in Fig. S2. $\dagger$ Their physical properties are listed in Table 1.

The solid state ${ }^{13} \mathrm{C}$ CP/MAS NMR spectra of all CMPs are displayed in Fig. 2. The typical chemical shifts between 110 and 150 ppm shown for all CMPs can be assigned to the aromatic rings in the polymer backbone. The signals from 80 to $100 \mathrm{ppm}$

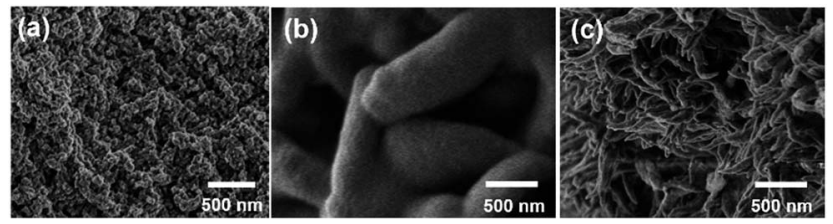

Fig. 1 SEM images of (a) BTh, (b) BThBT and (c) BBT.

Table 1 Physical properties of the designed CMPs

\begin{tabular}{lllll}
\hline CMP & $\begin{array}{l}S_{\text {BET }} \\
\left(\mathrm{m}^{2} \mathrm{~g}^{-1}\right)\end{array}$ & $\begin{array}{l}\text { Pore volume } \\
\left(\mathrm{cm}^{3} \mathrm{~g}^{-1}\right)\end{array}$ & $\begin{array}{l}\text { Pore size } \\
(\mathrm{nm})\end{array}$ & $\begin{array}{l}\text { Band gap } \\
(\mathrm{eV})\end{array}$ \\
\hline BTh & & & & \\
BThBT & 806 & 0.620 & 1.7 & 2.36 \\
BBT & 129 & 0.314 & 1.41 & 2.00 \\
& 129 & 0.631 & 1.7 & 2.12
\end{tabular}

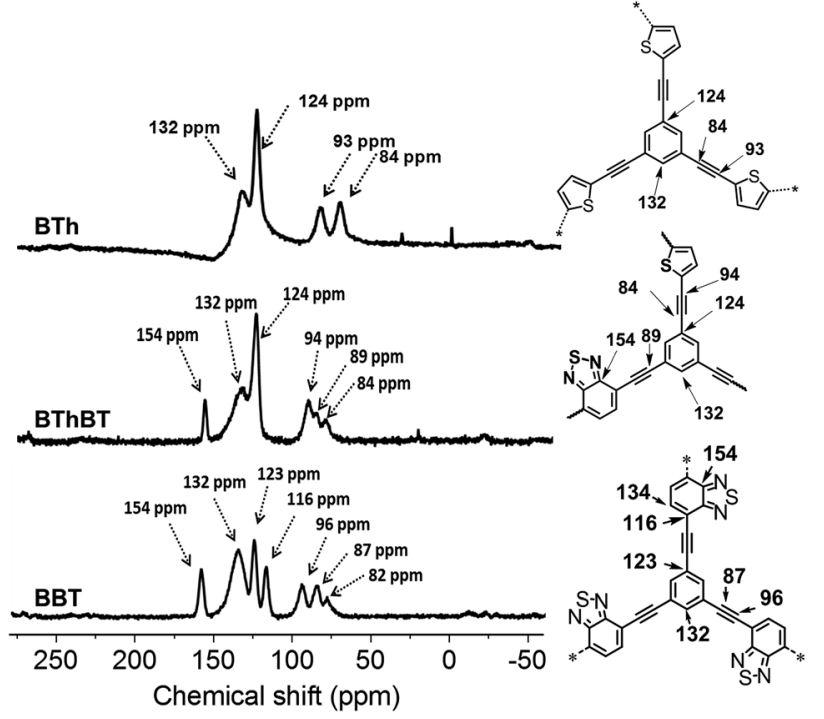

Fig. 2 Solid state ${ }^{13} \mathrm{C}$ CP/MAS NMR spectra of the CMPs. 
can be assigned to the carbon of the triple bond. A resonance peak at 155 ppm could be observed, which can be assigned to the adjacent carbon next to nitrogen in the BT unit. The thiophene (Th) unit can be characterized by the signal at about $124 \mathrm{ppm}$, which is assigned to the $=\mathrm{C}-\mathrm{S}$ single bond. ${ }^{36-38}$

The Fourier transform infrared (FTIR) spectra (Fig. 3a) showed a typical signal at $2300 \mathrm{~cm}^{-1}$ which could be assigned to the $-\mathrm{C} \equiv \mathrm{C}-$ stretching mode. ${ }^{39}$ The signals at $1570 \mathrm{~cm}^{-1}$ and $1480 \mathrm{~cm}^{-1}$ are characteristic of the $=\mathrm{N}-\mathrm{S}-$ and the $-\mathrm{C}=\mathrm{N}-$ stretching modes in the benzothiadiazole moiety. ${ }^{35}$ The vibrational peak at $790 \mathrm{~cm}^{-1}$ is attributed to the thiophene unit in the BTh network. ${ }^{37}$ The BThBT spectra contain signals corresponding to both the BT and Th units. ${ }^{40}$ Thermogravimetric analysis (TGA) indicated that BBT was thermally the most stable up to $300{ }^{\circ} \mathrm{C}$, whereas BTh and BThBT showed lower initial decomposition temperatures with BTh exhibiting the lowest weight loss among all of the CMPs (Fig. 3b).

Fig. 3c illustrates the UV/vis diffuse reflectance (DR) spectra of the CMPs. All three polymers showed a similar absorption range with maximal absorption between 300 and $450 \mathrm{~nm}$ and gradual levelling off until ca. $800 \mathrm{~nm}$. Additionally, a clear extension effect of the absorption range of the BT unit was revealed. The strong donor-based CMP, i.e. BTh, absorbs mainly in the blue light range. In comparison, the spectra of BThBT as well as those of BBT exhibited a gradual bathochromic shift up to $c a .60 \mathrm{~nm}$.

To gain further insight into the electronic properties, cyclic voltammetry (CV) measurements were conducted to reveal the energy band structure of the designed materials (Fig. S3 $\dagger$ ). The electronic band positions of the CMPs are displayed in Fig. 3d. BBT exhibited the highest LUMO level at $-0.92 \mathrm{~V} v s$. SCE among the CMPs, indicating its strong ability to activate molecular oxygen to its active states such as superoxide oxygen and singlet oxygen. The reduction potential of the $\mathrm{O}_{2} / \mathrm{O}_{2}{ }^{-}-$lies at $-0.57 \mathrm{~V}$ vs. SCE. ${ }^{41,42}$ The results strongly suggest the possible superior photocatalytic activity of BBT for oxidative reactions.
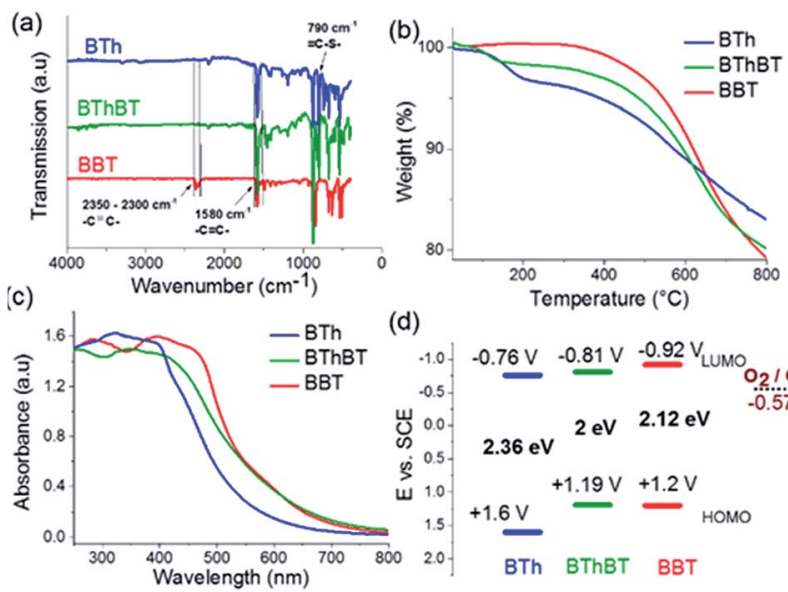

(d)

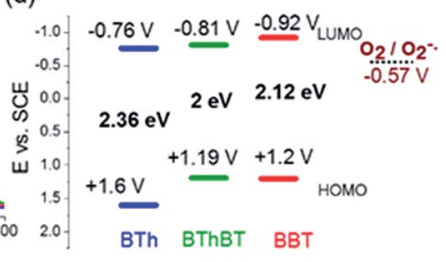

Fig. 3 (a) FTIR and (b) TGA spectra of the materials under a $\mathrm{N}_{2}$ atmosphere with a heating rate of $10{ }^{\circ} \mathrm{C} \mathrm{min}^{-1}$; (c) UV/vis DR spectra and (d) energy band structures of the as synthesized CMPs and the $\mathrm{O}_{2} / \mathrm{O}_{2} \cdot-$ couple.
To investigate the photocatalytic performance of the designed CMPs, we tested the oxidative cleavage of the $\mathrm{C}=\mathrm{C}$ bond using styrene as a model substrate under visible light irradiation. The experiments were first carried out at room temperature using BBT as the photocatalyst due to its highest reduction potential and molecular oxygen as an oxidant in various solvents. The results are listed in Table 2 . The conversion of styrene catalyzed by BBT was found to be strongly dependent on the solvent polarity. Benzaldehyde was mainly observed in various solvents, with relatively high selectivity (entries 1 to 8 , Fig. $\mathrm{S} 4 \dagger$ ). For instance, in an apolar solvent such as hexane (entry 1 in Table 2), a high selectivity of around $81 \%$ to benzaldehyde with a total styrene conversion of $7 \%$ was observed after $18 \mathrm{~h}$. Increasing the polarity of the solvent (ethanol, THF, nitromethane and acetonitrile) led to an increase of the conversion of styrene up to $44 \%$ with benzaldehyde yields ranging from $61 \%$ to $92 \%$ (entries 2, 3, 4 and 5 in Table 2).

When further increasing the solvent polarity by mixing water with acetonitrile, it was found that the photooxidation of styrene was noticeably enhanced upon addition of $\mathrm{H}_{2} \mathrm{O}$ to acetonitrile under blue light irradiation (entries 6,7 and 8 in Table 2). For example, the styrene conversion was increased by more than 2 -fold from $44 \%$ to $91 \%$, with a high benzaldehyde yield of about $86 \%$, when an optimized amount of water to acetonitrile $(\mathrm{v} / \mathrm{v}=1 / 24)$ was added (entry 8 in Table 2). These results shed light on the solvent-polarity-dependent-conversion on one hand and the effect of water on the acceleration of the photooxygenation of styrene by $\mathrm{O}_{2}$ on the other hand. ${ }^{43}$

The involvement of water in this reaction was further confirmed by the detection of 1-phenyl-1,2-ethanediol by GC-MS during the reaction, which is possibly due to the attack of water on the intermediate in the reaction process (Fig. S9†).

Taking into consideration the optimized reaction conditions above, the photocatalytic performance of the other designed CMPs was investigated under the same conditions using waterbased-biphasic systems $\left(\mathrm{MeCN} / \mathrm{H}_{2} \mathrm{O}: \mathrm{v} / \mathrm{v}=1 / 24\right)$. As listed in Table 2 (Fig. S5 $\dagger$ ), the reaction catalyzed by the BThBT reached a lower conversion of about $58 \%$ with only $43 \%$ yield to benzaldehyde, whereas BTh was not efficient for this reaction exhibiting only $16 \%$ of conversion with formation of benzaldehyde as the main product (entries 9 and 10). The higher photocatalytic efficiencies of the BT-containing CMPs, BBT and BThBT could be explained by a more efficient photogenerated charge separation within the polymer networks due to the donor-acceptor combinations. In comparison, BTh only contains electron donor units, which inhibit an efficient charge separation and transfer inside the CMP. Indeed, Fig. 4a reveals the most intense photocurrent signal of BBT among the three CMPs under visible light, indicating that the photogenerated charge separation in BBT is more efficient than that in BThBT and BTh.

To further investigate the reaction mechanism and reveal the specific role of the photogenerated electron-hole pairs during the photocatalytic process, we conducted a number of control experiments, using BBT as the photocatalyst. The results are also listed in Table 2 (Fig. S6†). In the absence of light and molecular oxygen, no reaction conversion was determined 
Table 2 Photocatalytic $\mathrm{C}=\mathrm{C}$ bond cleavage using various CMPs with various reaction conditions

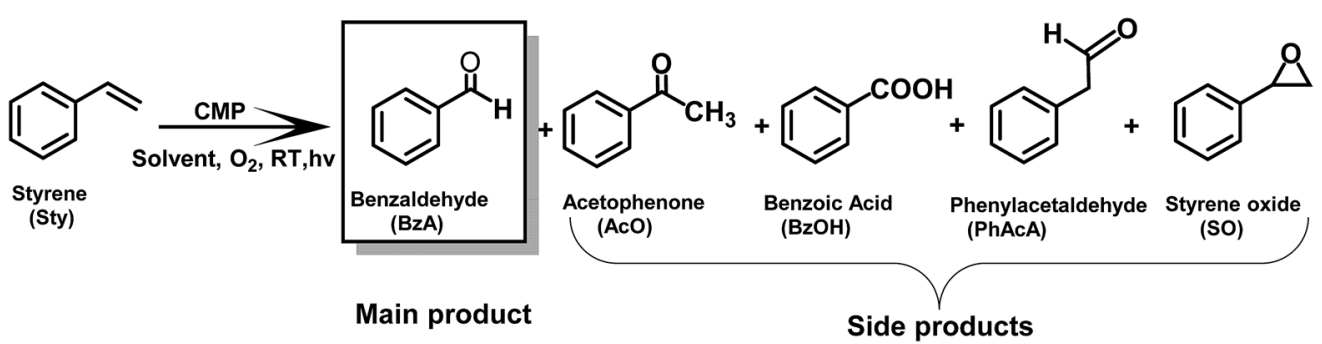

\begin{tabular}{|c|c|c|c|c|c|c|c|}
\hline Entry $^{a}$ & Photocatalyst & Solvent & $\mathrm{O}_{2}$ & Light & Additives & $\begin{array}{l}\text { Conversion }^{b} \\
(\%)\end{array}$ & $\begin{array}{l}\text { Selectivity } \\
\text { of } \mathrm{BzA}^{b}(\%)\end{array}$ \\
\hline 1 & BBT & Hexane & + & + & - & 7 & 81 \\
\hline 2 & BBT & Ethanol & + & + & - & 15 & 85 \\
\hline 3 & BBT & THF & + & + & - & 23 & 92 \\
\hline 4 & BBT & $\mathrm{MeNO}_{2}$ & + & + & - & 43 & 62 \\
\hline 5 & BBT & $\mathrm{MeCN}$ & + & + & - & 44 & 60 \\
\hline 6 & BBT & $\mathrm{MeCN} / \mathrm{H}_{2} \mathrm{O}(\mathrm{v} / \mathrm{v}=24 / 1)$ & + & + & - & 59 & 57 \\
\hline 7 & BBT & $\mathrm{MeCN} / \mathrm{H}_{2} \mathrm{O}(\mathrm{v} / \mathrm{v}=1 / 1)$ & + & + & - & 77 & 51 \\
\hline 8 & BBT & $\mathrm{MeCN} / \mathrm{H}_{2} \mathrm{O}(\mathrm{v} / \mathrm{v}=1 / 24)$ & + & + & - & 91 & 85 \\
\hline 9 & BTh & $\mathrm{MeCN} / \mathrm{H}_{2} \mathrm{O}(\mathrm{v} / \mathrm{v}=1 / 24)$ & + & + & - & 16 & 69 \\
\hline 10 & BThBT & $\mathrm{MeCN} / \mathrm{H}_{2} \mathrm{O}(\mathrm{v} / \mathrm{v}=1 / 24)$ & + & + & - & 58 & 43 \\
\hline $11^{c}$ & BBT & $\mathrm{MeCN} / \mathrm{H}_{2} \mathrm{O}(\mathrm{v} / \mathrm{v}=1 / 24)$ & + & - & - & n.d & n.d \\
\hline $12^{d}$ & BBT & $\mathrm{MeCN} / \mathrm{H}_{2} \mathrm{O}(\mathrm{v} / \mathrm{v}=1 / 24)$ & - & + & - & n.d & n.d \\
\hline $13^{e}$ & - & $\mathrm{MeCN} / \mathrm{H}_{2} \mathrm{O}(\mathrm{v} / \mathrm{v}=1 / 24)$ & + & + & - & 48 & 79 \\
\hline $14^{f}$ & - & $\mathrm{MeCN}$ & + & + & - & 30 & 85 \\
\hline $15^{g}$ & BBT & $\mathrm{MeCN} / \mathrm{H}_{2} \mathrm{O}(\mathrm{v} / \mathrm{v}=1 / 24)$ & + & + & $\mathrm{KI}$ as a hole scavenger & 2 & 100 \\
\hline $16^{h}$ & BBT & $\mathrm{MeCN} / \mathrm{H}_{2} \mathrm{O}(\mathrm{v} / \mathrm{v}=1 / 24)$ & + & + & $\mathrm{NaN}_{3}$ as a singlet oxygen scavenger & 17 & 69 \\
\hline $17^{i}$ & BBT & $\mathrm{MeCN} / \mathrm{H}_{2} \mathrm{O}(\mathrm{v} / \mathrm{v}=1 / 24)$ & + & + & Isopropanol as a hydroxyl radical scavenger & 88 & 45 \\
\hline $18^{j}$ & BBT & $\mathrm{MeCN} / \mathrm{H}_{2} \mathrm{O}(\mathrm{v} / \mathrm{v}=1 / 24)$ & + & + & Benzoquinone as a superoxide scavenger & 1 & 100 \\
\hline
\end{tabular}

${ }^{a}$ Standard reaction conditions: $0.1 \mathrm{mmol}$ of styrene, $10 \mathrm{mg}$ of CMP in $1.5 \mathrm{~mL}$ of solvent under the irradiation of a blue LED lamp (460 $\mathrm{nm}$, $0.16 \mathrm{~W}$

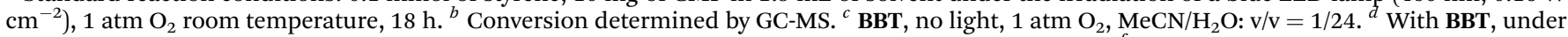
light, no $\mathrm{O}_{2}, \mathrm{MeCN} / \mathrm{H}_{2} \mathrm{O}: \mathrm{v} / \mathrm{v}=1 / 24 .{ }^{e}$ Without catalyst, under light, $1 \mathrm{~atm} \mathrm{O}_{2}, \mathrm{MeCN} / \mathrm{H}_{2} \mathrm{O} \mathrm{v} / \mathrm{v}=1 / 24 .{ }^{f}$ Without catalyst, under light, 1 atm $\mathrm{O}_{2}$, acetonitrile. ${ }^{g} \mathrm{KI}$ as a hole scavenger. ${ }^{h} \mathrm{NaN}_{3}$ as a singlet oxygen scavenger. ${ }^{i}$ Isopropanol as a hydroxyl radical scavenger. ${ }^{j}$ Benzoquinone (BQ) as a superoxide scavenger.

(entries 11 and 12 in Table 2). Without using a photocatalyst, only a conversion of 30-42\% was determined (entries 13 and 14 in Table 2). The results indicate that all three components are indispensable. The effect of specific scavengers was also studied.

A conversion of $2 \%$ was obtained in the presence of $\mathrm{KI}$ as a hole scavenger (entry 15 in Table 2). A conversion of $17 \%$ was reached in the presence of $\mathrm{NaN}_{3}$ as a singlet oxygen scavenger (entry 16 in Table 2). Use of isopropanol as a hydroxyl scavenger led to a conversion of $88 \%$ (entry 17 in Table 2). Significantly, a conversion of only $1 \%$ was determined in the presence of benzoquinone as a superoxide scavenger (entry 18 in Table 2). These results indicate that the activated oxygen species (superoxide and singlet oxygen) and photogenerated hole all participate during the oxidative $\mathrm{C}=\mathrm{C}$ bond cleavage reaction. To precisely analyze the possible active oxygen species (superoxide and singlet oxygen) and their exact contribution during the photocatalytic reaction, we further conducted electron spin resonance (EPR) experiments using 5,5-dimethyl-1-pyrroline $N$-oxide (DMPO) and 2,2,6,6-tetramethylpiperidine (TEMP) as superoxide and singlet oxygen trapping agents, respectively. As shown in Fig. $4 \mathrm{~b}$ and c, both active oxygen species could be determined, confirming their active roles in the reaction.

Based on the observations drawn from the experiments described above, we suggest a modified reaction mechanism for the oxidative $\mathrm{C}=\mathrm{C}$ bond cleavage and aldehyde formation inspired from the literature ${ }^{\mathbf{1 3 , 4 3}}$ (Fig. 5). Under visible light irradiation, the charge separation occurs within the CMP based photocatalyst. The photogenerated hole of BBT oxidizes styrene $\mathbf{1}$ into its cationic radical intermediate 1a. Further proof of the formation of the radical intermediate could be observed using $N$-tert-butyl- $\alpha$-phenylnitrone (PNB) as a radical trapping agent (Fig. 4d). A typical pattern for the PBN trapped radical with $g$ value equal to 2.00667 has been recorded.

In the same time, the electron from the LUMO of the CMP assisted the reductive activation of molecular oxygen into superoxide $\left(\mathrm{O}_{2}={ }^{-}\right)$, which undergoes $[2+2]$ cycloaddition with the cationic radical of styrene to generate the 3-phenyl-1,2dioxetane 2 . Note that, singlet oxygen $\left({ }^{1} \mathrm{O}_{2}\right)$ also took part in the oxidation step. 1-Phenyl-1,2-ethanediol 3 is then formed due to the attack of the water on the dioxetane intermediate, which ultimately is cleaved into benzaldehyde $\mathbf{4}$, releasing 


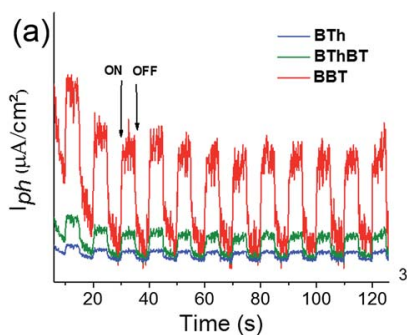

(b)

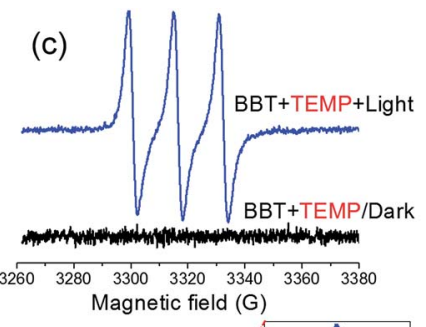

(d)

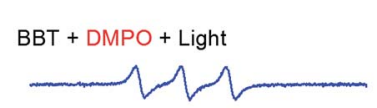

BBT + DMPO in Dark

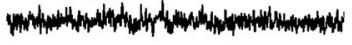
$\begin{array}{lllllllllllll}3260 & 3280 & 3300 & 3320 & 3340 & 3360 & 33803260 & 3280 & 3300 & 3320 & 3340 & 3360 & 3380 \\ & & \text { Magnetic field (G) } & & & & & & \text { Magnetic field (G) }\end{array}$

Magnetic field (G)

Fig. 4 (a) Photocurrent responses of the CMPs under the illumination of visible light with a wavelength $\geq 420 \mathrm{~nm}$ with a light ON/OFF model. EPR spectra of (b) DMPO- $\mathrm{O}_{2}=-(g=2.00639)$ and (c) $\mathrm{TEMP}^{-}{ }^{1} \mathrm{O}_{2}$ adducts ( $g=2.00645$ ) using BBT as the photocatalyst in the dark and under light irradiation $\left(\lambda=460 \mathrm{~nm}, 0.065 \mathrm{~W} \mathrm{~cm}^{-2}\right)$. (d) EPR spectra using PBN as a radical trapping agent for the radical intermediate of styrene, with BBT, under $\mathrm{O}_{2}$ and light irradiation ( $\left.g=2.00667\right)$.

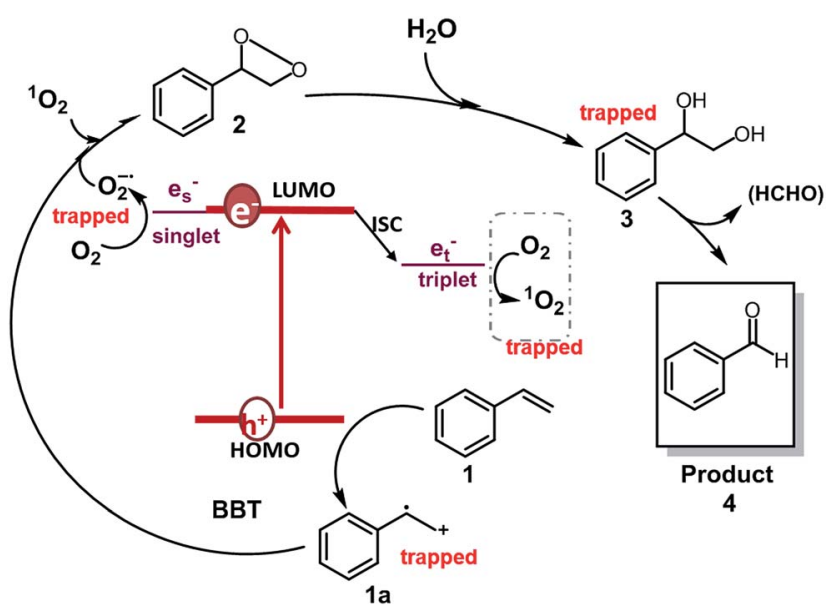

Fig. 5 Suggested reaction mechanism for the aerobic $C=C$ double bond cleavage of styrene using BBT as the photocatalyst.

formaldehyde as the possible side product. High-performance liquid chromatography (HPLC) did not give a confirmation of the existence of formaldehyde. Nevertheless, the ${ }^{1} \mathrm{H}$ NMR spectra showed traces of possible formaldehyde formation (Fig. S7 $\dagger$ ). The results indicate that the formed formaldehyde as the side product could be directly oxidized. Additionally, the overoxidation of the aldehyde may result in the formation of carboxylic acids, depending on the nature of the substrate (see the scope of substrates below).

To further demonstrate the general applicability of BBT as a photocatalyst, screening experiments with various styrene derivatives have been carried out under the same conditions and the results are illustrated in Fig. 6. High conversion was

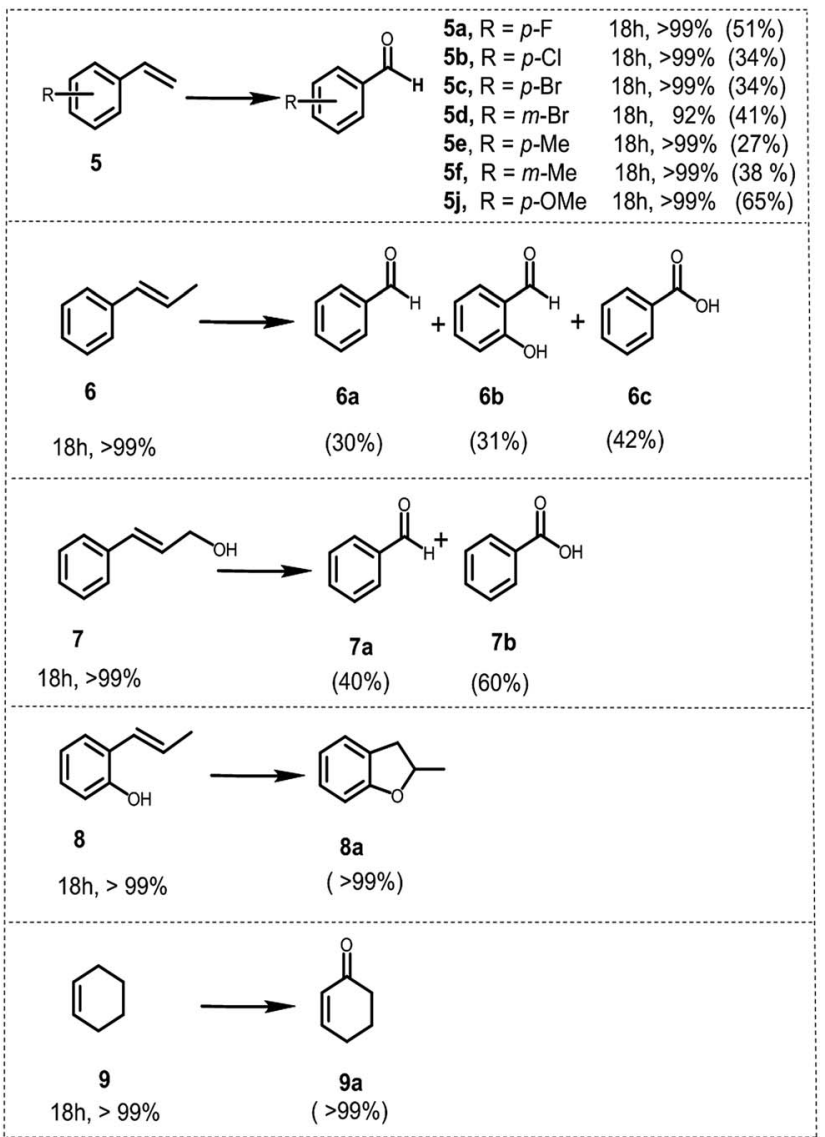

Fig. 6 Scope of alkenes for the photocatalytic $\mathrm{C}=\mathrm{C}$ bond cleavage using BBT as the photocatalyst.

obtained in most cases under the same conditions. However, both electron-withdrawing substitution groups on the phenyl rings of the substrates such as $-\mathrm{F}(\mathbf{5 a})$ or $-\mathrm{Cl}(\mathbf{5 b})$ or $-\mathrm{Br}(\mathbf{5 c}$ and 5d) and electron-donating substitution groups such as methyl (5e and 5f) and methoxy (5j) led to lower benzaldehyde yield than the model reaction. Nevertheless, it appears that the variation of the position of the substituent on the phenyl ring does not have considerable effect on the conversion and on the yield of the corresponding aldehyde, either.

Following the same procedure, trans- $\beta$-methyl styrene 6 and cinnamyl alcohol 7 were utilized as substrates to test secondary olefins under identical conditions. Both 6 and 7 were oxidized to the corresponding carbonyl compounds, offering benzoic acids as the main product with a yield of $40 \%$ (6c) and $60 \%$ (7b) respectively, which implied that BBT was also suitable for the secondary olefins. Additionally, BBT could be efficiently used for the synthesis of benzofuran 8a from 2-allylphenol 8 and 2cyclohexen-1-one 9a from cyclohexene 9, both in excellent yields.

The stability and reusability of BBT were investigated via 5 additional repeat experiments under the same reaction conditions. As shown in Fig. S8, $\uparrow$ BBT could catalyze the reaction for 5 additional cycles without significantly affecting its catalytic efficiency. However, the yield to benzaldehyde decreased in favor of the formation of 1-phenyl-1,2-ethanediol (compound 3 
in Fig. 5). This indicates a slowing-down of the photocatalytic reaction after five cycles of use. FT-IR and UV/vis DR spectra (Fig. S9 $\dagger$ ) remain unchanged after the repeat experiments, which further confirm the high stability of the photocatalyst.

\section{Conclusions}

In summary, we demonstrated the design strategy of conjugated microporous polymers (CMPs) as heterogeneous, visible-light active and stable photocatalysts for the photocatalytic $\mathrm{C}=\mathrm{C}$ bond cleavage reaction of alkenes. By combining specific electron donor and acceptor building blocks with the CMP backbone structure, we found out that the CMP containing benzothiodiazole as a strong electron acceptor and phenyl as a weak electron donor was the most efficient photocatalyst. The photocatalytic cleavage reaction of the $\mathrm{C}=\mathrm{C}$ bond was found to be strongly dependent on the solvent polarity, with the aqueous reaction mixture being the ideal reaction medium with benzaldehydes as main products with high efficiency and selectivity.

\section{Experimental procedure}

\section{Materials}

Copper(I) iodide (99\%), tetrakis(triphenylphosphine)palladium(0) (99\%), p-benzoquinone (98\%), sodium azide (99\%), potassium iodide (>99.9\%), triethylamine (99\%) and 2,5-dibromothiophene (96\%), were purchased from Sigma Aldrich. 1,3,5Triethynylbenzene (>98\%) and 4,4-dibromobiphenyl (98\%) were purchased from Alfa Aesar (Massachusetts, USA). 4,7-Dibromo2,1,3-benzothiadiazole (97\%) (Br-BT) was purchased from COMBI-BLOCKS. Isopropanol (99.5\%) and anhydrous $\mathrm{N}, \mathrm{N}$ dimethylformamide (99.8\%) were purchased from Acros organics. Acetonitrile was purchased from Fischer Chemical. Styrene and styrene derivatives were purified by elution through neutral aluminium oxide (50-200 $\mu \mathrm{m})$ and anhydrous $\mathrm{CaCl}_{2}$ (w/w, 95/5) in order to remove peroxides and other impurities. Chemicals and solvents purchased from commercial sources were used as received unless otherwise noted.

\section{Methods}

X-ray diffraction (XRD) patterns were obtained using a Philips PW1050 diffractometer with $\mathrm{CuK} \alpha$ radiation at $k=1.5418 \AA$. UV/vis absorption spectra were measured using a Perkin-Elmer Lambda 25 UV-Vis spectrometer. FTIR spectra were obtained using a FTIR spectrophotometer TENSOR II Bruker equipped with a platinum ATR. Solid state ${ }^{13} \mathrm{C}$ CP/MAS NMR measurements were performed with a standard $4 \mathrm{~mm}$ magic angle spinning MAS double resonance probe head at $300 \mathrm{MHz}$ Larmor frequency. TGA measurements were carried out on a Mettler Toledo TGA-851 system. The temperature was increased from $25{ }^{\circ} \mathrm{C}$ to $800{ }^{\circ} \mathrm{C}\left(10{ }^{\circ} \mathrm{C} \mathrm{min}^{-1}\right)$ under a nitrogen atmosphere. Electron paramagnetic resonance (EPR) spectroscopy was performed using a Magnettech MS 200 spectrometer. The nitrogen sorption data were measured to determine the surface area and the pore size distribution; the data were recorded at $77.3 \mathrm{~K}$ on an Autosorb 1 using QuadraWin Software (Quantchrome instruments). CV and photocurrent measurements were conducted using an Autolab Potentiostat PGSTAT204 (Metrohm). A glassy carbon working electrode, a Pt counter electrode and a SCE reference electrode were used. The SEM images were collected using a LEO Gemini 1530 (Carl Zeiss AG, Germany) with an in-lens SE detector.

\section{Synthesis of BTh, BThBT and BBT}

The synthesis of BBT, BThBT and BTh was performed via palladium catalyzed Sonogashira-Hagihara cross coupling reactions. The cross-linker 1,3,5-triethynylbenzene $(150 \mathrm{mg}$, $1 \mathrm{mmol}, 1$ equiv.), tetrakis(triphenylphosphine)palladium(0) (35 mg, $0.03 \mathrm{mmol}, 0.03$ equiv.) and copper(I) iodide $(5.7 \mathrm{mg}$, $0.03 \mathrm{mmol}, 0.03$ equiv.) were dissolved in $20 \mathrm{~mL}$ of $\mathrm{DMF} /$ triethylamine mixture with a volume ratio of $1: 1$. Then, the co-monomer 4,7-dibromo-2,1,3-benzothiadiazole (294 mg, 1 mmol, 1 equiv.) was added for BBT, and the co-monomer 2,5dibromothiophene (119 $\mu \mathrm{L}, 1 \mathrm{mmol}, 1$ equiv.) was added for BTh. As for the BThBT, both co-monomers (4,7-dibromo-2,1,3benzothiadiazole and 2,5-dibromothiophene) were added in an equal ratio. The reaction mixtures were degassed with $\mathrm{N}_{2}$ for $10 \mathrm{~min}$. After the degassing process, the reactive mixtures were heated at $80{ }^{\circ} \mathrm{C}$ for $48 \mathrm{~h}$. After cooling, the precipitated powders were washed with methanol, acetone and water several times. The crude products were then extracted in a Soxhlet extractor for $24 \mathrm{~h}$ using a mixture of dichloromethane and methanol. Finally, the products were dried overnight at $60{ }^{\circ} \mathrm{C}$ under vacuum. Yield $($ BTh $)=52 \%$, yield $($ BThBT $)=64 \%$ and yield $($ BBT $)=55 \%$.

Metal residue analysis. BBT: $\operatorname{ICP}(\mathrm{Pd})=3.75 \mathrm{ppm}, \operatorname{ICP}(\mathrm{Cu})=$ 0.05 ppm; BThBT: $\mathrm{ICP}(\mathrm{Pd})=10 \mathrm{ppm}, \operatorname{ICP}(\mathrm{Cu})=0.22 \mathrm{ppm}$; BTh: $\operatorname{ICP}(\mathrm{Pd})=3.77 \mathrm{ppm} \operatorname{ICP}(\mathrm{Cu})=$ n.d. (not determined).

\section{General procedure for the $\mathrm{C}=\mathrm{C}$ bond cleavage using CMPs as photocatalysts}

$0.1 \mathrm{mmol}$ of styrene or its derivatives or alkenes and $10 \mathrm{mg}$ of the photocatalyst were added to $1.5 \mathrm{~mL}$ of solvent in a glass vial equipped with an oxygen balloon. A blue LED lamp (460 nm, $0.065 \mathrm{~W} \mathrm{~cm}^{-2}$, OSA Opto Light $\mathrm{GmbH}$ ) was used as the light source. The reaction mixture was stirred at room temperature and under irradiation for $18 \mathrm{~h}$. The conversion and selectivity were determined via GC-MS. The GCMS spectra are displayed in the ESI. For the repeat experiments, the CMP was filtered and washed several times with methanol, $\mathrm{CH}_{2} \mathrm{Cl}_{2}$ and acetone, and then dried under vacuum before being used for the repeat experiments.

\section{Conflicts of interest}

There are no conflicts to declare.

\section{Acknowledgements}

K. A. I. Z. acknowledges the Max Planck Society for financial support. C. A. is a recipient of a fellowship through funding of the Excellence Initiative (DFG/GSC 266) of the Graduate School of Excellence "MAINZ" (Materials Science in Mainz). Open Access funding was provided by the Max Planck Society. 


\section{Notes and references}

1 V. Kogan, M. M. Quintal and R. Neumann, Org. Lett., 2005, 7, 5039-5042.

2 B. Liu, P. Wang, A. Lopes, L. Jin, W. Zhong, Y. Pei, S. L. Suib and J. He, ACS Catal., 2017, 7, 3483-3488.

3 A. P. S. Oliveira, I. S. Gomes, A. S. B. Neto, A. C. Oliveira, J. M. Filho, G. D. Saraiva, J. M. Soares and S. TehuacaneroCuapa, Mol. Catal., 2017, 436, 29-42.

4 A. Rubinstein, P. Jiménez-Lozanao, J. J. Carbó, J. M. Poblet and R. Neumann, J. Am. Chem. Soc., 2014, 136, 10941-10948.

5 B. Wang, J. Zhang, X. Zou, H. Dong and P. Yao, Chem. Eng. J., 2015, 260, 172-177.

6 B. Liu, F. Jin, T. Wang, X. Yuan and W. Han, Angew. Chem., Int. Ed., 2017, 56, 12712-12717.

7 A. B. Khemnar and B. M. Bhanage, RSC Adv., 2014, 4, 89398942.

8 A. P. S. Oliveira, I. S. Gomes, A. C. Oliveira, J. M. Filho, G. D. Saraiva, J. M. Soares, F. F. de Sousa and A. Campos, Catalysts, 2017, 7, 323.

9 B. Feng, Z. Hou, X. Wang, Y. Hu, H. Li and Y. Qiao, Green Chem., 2009, 11, 1446-1452.

10 Z. Zhang, Y. Kumamoto, T. Hashiguchi, T. Mamba, H. Murayama, E. Yamamoto, T. Ishida, T. Honma and M. Tokunaga, ChemSusChem, 2017, 10, 3482-3489.

11 Y. Guo, L. Zhengwang, W. Guangjian, Y. Huang and F. Kang, Appl. Surf. Sci., 2011, 258, 1082-1090.

12 D. Shen, C. Saracini, Y.-M. Lee, W. Sun, S. Fukuzumi and W. Nam, J. Am. Chem. Soc., 2016, 138, 15857-15860.

13 A. K. Singh, R. Chawla and L. D. S. Yadav, Tetrahedron Lett., 2015, 56, 653-656.

14 K. Zhang, D. Kopetzki, P. H. Seeberger, M. Antonietti and F. Vilela, Angew. Chem., Int. Ed., 2013, 52, 1432-1436.

15 H. Urakami, K. Zhang and F. Vilela, Chem. Commun., 2013, 49, 2353-2355.

16 S. Ghasimi, S. A. Bretschneider, W. Huang, K. Landfester and K. A. I. Zhang, Adv. Sci., 2017, 4, 1700101.

17 Y. Li, M. Liu and L. Chen, J. Mater. Chem. A, 2017, 5, 1375713762.

18 X. Ding and B.-H. Han, Angew. Chem., Int. Ed., 2015, 54, 6536-6539.

19 Z. J. Wang, S. Ghasimi, K. Landfester and K. A. I. Zhang, Chem. Commun., 2014, 50, 8177-8180.

20 J.-X. Jiang, Y. Li, X. Wu, J. Xiao, D. J. Adams and A. I. Cooper, Macromolecules, 2013, 46, 8779-8783.

21 Z. J. Wang, S. Ghasimi, K. Landfester and K. A. Zhang, Adv. Mater., 2015, 27, 6265-6270.

22 N. Kang, J. H. Park, K. C. Ko, J. Chun, E. Kim, H. W. Shin, S. M. Lee, H. J. Kim, T. K. Ahn, J. Y. Lee and S. U. Son, Angew. Chem., Int. Ed., 2013, 52, 6228-6232.

23 Z. J. Wang, S. Ghasimi, K. Landfester and K. A. I. Zhang, J. Mater. Chem. A, 2014, 2, 18720-18724.
24 W. Huang, B. C. Ma, D. Wang, Z. J. Wang, R. Li, L. Wang, K. Landfester and K. A. I. Zhang, J. Mater. Chem. A, 2017, 5, 3792-3797.

25 J. Luo, X. Zhang and J. Zhang, ACS Catal., 2015, 5, 22502254.

26 Z. J. Wang, K. Landfester and K. A. I. Zhang, Polym. Chem., 2014, 5, 3559-3562.

27 S. Dadashi-Silab, H. Bildirir, R. Dawson, A. Thomas and Y. Yagci, Macromolecules, 2014, 47, 4607-4614.

28 R. Li, B. C. Ma, W. Huang, L. Wang, D. Wang, H. Lu, K. Landfester and K. A. I. Zhang, ACS Catal., 2017, 7, 30973101.

29 V. R. Battula, H. Singh, S. Kumar, I. Bala, S. K. Pal and K. Kailasam, ACS Catal., 2018, 8, 6751-6759.

30 W. Zhang, J. Tang, W. Yu, Q. Huang, Y. Fu, G.-C. Kuang, C. Pan and G. Yu, ACS Catal., 2018, 8, 8084-8091.

31 R. S. Sprick, J.-X. Jiang, B. Bonillo, S. Ren, T. Ratvijitvech, P. Guiglion, M. A. Zwijnenburg, D. J. Adams and A. I. Cooper, J. Am. Chem. Soc., 2015, 137, 3265-3270.

32 C. Yang, B. C. Ma, L. Zhang, S. Lin, S. Ghasimi, K. Landfester, K. A. I. Zhang and X. Wang, Angew. Chem., Int. Ed., 2016, 55, 9202-9206.

33 R. S. Sprick, C. M. Aitchison, E. Berardo, L. Turcani, L. Wilbraham, B. M. Alston, K. E. Jelfs, M. A. Zwijnenburg and A. I. Cooper, J. Mater. Chem. A, 2018, 6, 11994-12003.

34 L. Wang, Y. Wan, Y. Ding, S. Wu, Y. Zhang, X. Zhang, G. Zhang, Y. Xiong, X. Wu, J. Yang and H. Xu, Adv. Mater., 2017, 29, 1702428.

35 C. Ayed, W. Huang, R. Li, L. C. da Silva, D. Wang, O. Suraeva, W. Najjar and K. A. I. Zhang, Part. Part. Syst. Charact., 2018, 35, 1700234.

36 B. C. Ma, S. Ghasimi, K. Landfester and K. A. I. Zhang, J. Mater. Chem. B, 2016, 4, 5112-5118.

37 B. C. Ma, S. Ghasimi, K. Landfester, F. Vilela and K. A. I. Zhang, J. Mater. Chem. A, 2015, 3, 16064-16071.

38 R. Li, B. C. Ma, W. Huang, L. Wang, D. Wang, H. Lu, K. Landfester and K. A. I. Zhang, ACS Catal., 2017, 7, 30973101.

39 Z. J. Wang, K. Garth, S. Ghasimi, K. Landfester and K. A. I. Zhang, ChemSusChem, 2015, 8, 3459-3464.

40 R.-M. Latonen, C. Kvarnström and A. Ivaska, J. Electroanal. Chem., 2001, 512, 36-48.

41 S. Ghosh, N. A. Kouame, S. Remita, L. Ramos, F. Goubard, P.-H. Aubert, A. Dazzi, A. Deniset-Besseau and H. Remita, Sci. Rep., 2015, 5, 18002.

42 R. Li, Z. J. Wang, L. Wang, B. C. Ma, S. Ghasimi, H. Lu, K. Landfester and K. A. I. Zhang, ACS Catal., 2016, 6, 11131121.

43 Y. Ren, Y. Che, W. Ma, X. Zhang, T. Shen and J. Zhao, New J. Chem., 2004, 28, 1464-1469. 